Scott, N., \& Baggio, R. (2016). Network, Tourism. In J. Jafari \& H. Xiao (Eds.), Encyclopaedia of Tourism: Springer.

\title{
Network, tourism
}

A tourism system is complex and dynamic but may be described as a network providing a means to represent the interactions among its different components. In general, a network is a picture of the connections or relationships among a set of objects (people, hence stakeholder network or social network; organizations, hence organizational network; aviation routes, hence route network). Formal analysis of networks (or network analysis) began in the mathematical field of graph theory, a branch of topology. A graph is an abstract mathematical object composed of nodes (vertices, actors) connected by relationships (links, edges) and can be represented by a matrix (adjacency matrix), thus allowing the use of linear algebra analysis methods.

These methods were applied in sociology to the study of social networks and the relations among sets of people (actors), predicated on the belief that a person's behavior is affected by that person's position and the structural properties of the network. Examples of relationship links that can be represented as a social network include communication, friendship, or economic transactions. Pairwise relationships among actors can be arrayed in a table (called a sociomatrix) and the network diagram drawn from this information is often referred to as a sociogram. Sociomatrices and sociograms feature centrally in sociometry, an elaborate but simple form of analysis pioneered by Moreno in the 1940s and used to study social groups. Recently, physicists and mathematicians, stimulated by developments in information technology, have studied complex networks using tools from statistical physics (Newman 2010).

Such studies may examine the network of a single object (egocentric network) and how that object's position may affect its behaviour, the characteristics of all the participants in the network (systemic network) such as in the work by Granovetter (1973), or the flow of information within a network, as in the process of innovation, or the establishment of synchronized opinions. Baggio, Scott and Cooper (2010) provide an overview of the field as a whole. Important properties of a network include cohesion (calculated by the number of actual links/number of possible links if the network is fully connected), centrality (various measures are used: betweenness centrality calculates how many times a node is a bridge on 
the shortest path between two other nodes), and connections bridging different cliques (important in knowledge transfer). Furthermore, numerical simulations have been widely used to uncover structural characteristics, such as modular or hierarchical organization, or to study the unfolding of dynamic processes such as information and knowledge diffusion, consensus formation, or resilience (capability to withstand external shocks) (Barrat, Barthélémy and Vespignani 2008; Newman 2010). Resilience is increased through adding redundant links between critical nodes.

\section{Stakeholder networks and data collection}

Early tourism studies examined inter-organizational networks to determine the effectiveness of coordination of cooperative marketing associations (Palmer and Bejou 1995), in the same way that political scientists studied policy networks (Dredge 2006; Rhodes 1990). Tourism is a networked industry where loose clusters of organizations within a destination, as well as networks of cooperative and competitive organizations linking destinations, cooperate and compete in a process of dynamic evolution. A mature destination may contain a central group of powerful stakeholders surrounded by a diffuse cloud of less well connected organizations, forming a core-periphery structure.

Destinations can be thought of as networks of enterprises, governments, and other organizations. Studies conducted so far have highlighted the complex nature of the destination networks examined. Researchers have also demonstrated the hierarchical composition of destinations by investigating the formation and composition of communities, finding quite varied patterns, different from the usual subdivision in terms of business types or geographical proximity. Knowledge and opinion diffusion within networks have been modeled numerically, suggesting the possibility of finding methods to optimize the network with respect to these processes, typically by creating new connections or rearranging them in order to favor the formation of communities. Collaborative and cooperative practices have been associated with some structural characteristics of the networks, primarily the existence of well-defined modular structures or the presence of high clustering of the links.

Data collection for a network study must be performed carefully, and conducted in a way that guarantees the highest completeness of the data (nodes and links) for the network being examined. In fact, the literature has highlighted a number of unexpected and antiintuitive outcomes, mainly due to the high skewedness of the different properties that network elements possess. As a result, standard statistical methods mostly based on parametric analysis may not be applied to network data. For the same reason, pure qualitative 
investigations, when not verified by a quantitative analysis, risk misinterpreting important features.

\section{Future developments}

Network studies in tourism are still at an early stage. A wider collection of cases is needed in order to better understand similarities and differences in structural characteristics. Moreover, longitudinal (in time) studies are required for being able to formulate more rigorous evolutionary models. Finally, deeper qualitative analyses are needed in order to interpret the quantitative outcomes of the analyses conducted, and more quantitative studies are needed to support many qualitative outcomes of tourism systems’ studies.

See also: Actor-network theory, information technology, social network analysis, partnership.

\section{References}

Baggio, R., N., Scott, and C. Cooper 2010 Network Science: A Review Focused on Tourism. Annals of Tourism Research 37:802-827.

Barrat, A., M., Barthélémy, and A. Vespignani 2008 Dynamical Processes on Complex Networks. Cambridge: Cambridge University Press.

Dredge, D.

2006 Policy Networks and the Local Organisation of Tourism. Tourism Management 27:269-280.

Granovetter, M.

1973 The Strength of Weak Ties. American Journal of Sociology 78:1360-1380.

Newman, $\mathrm{M}$.

2010 Networks: An Introduction. Oxford: Oxford University Press.

Palmer, A., and D. Bejou

1995 Tourism Destination Marketing Alliances. Annals of Tourism Research 22:616629.

Rhodes, R.

1990 Policy Networks: A British Perspective. Journal of Theoretical Politics 2:293317. 
Noel Scott

Griffith University, Australia

noel.scott@griffith.edu.au

Rodolfo Baggio

Bocconi University Italy rodolfo.baggio@unibocconi.it 